\title{
La innovación financiera empresarial caracterizada por los índices de gestión e indicadores convencionales
}

\author{
Laureano Triana Rubio ${ }^{1}$
}

Recibido: 14 de mayo de 2016

Aprobado: 17 de junio de 2016

\begin{abstract}
Triana L., (2016). La innovación financiera empresarial caracterizada por los índices de gestión e indicadores convencionales. Activos, 26, 185-204.
\end{abstract}

Clasificación JEL: G30, O16

\section{Resumen}

Este artículo, producto de investigación, analiza los cambios en las prácticas y en los procedimientos empresariales a partir del Manual de Oslo sobre innovación, consistente en la implementación de sistemas adecuados de información y medición. En los últimos años han surgido diferentes iniciativas de mejora en cuanto al logro de desempeño eficiente y la creación de valor; el propósito especial de esta investigación es proponer un modelo de sistema sostenible basado en índices de gestión e indicadores convencionales,

1 Docente, investigador y profesor emérito: Universidad Autónoma del Caribe. Contador público. Especialista en Finanzas y Gestión Contable. Líder del grupo de investigación ERCONFI. Cód. 043585 de COLCIENCIAS. Autor de los libros Valoración de empresas mediante el análisis de flujos de caja (ISBN-978-958-8524-23-8) y Modelo de gestión administrativa, contable, financiera y de auditoría para instituciones educativas. Construcción del PEI. (ISBN-978-958-8524-64-1). Correo electrónico: LTriana@uac.edu.co 
como resultado del estudio adelantado en empresas con mayor impacto socioeconómico en la ciudad de Barranquilla y la región Caribe.

\title{
Palabras clave
}

Gestión, impacto, innovación, Manual de Oslo, sostenible.

Triana L., (2016). Financial innovation of enterprises characterized by the management and conventional index. Activos, 26, 185-204.

\begin{abstract}
This article product research analyzes the changes in business practices and procedures, from Oslo Manual on innovation, namely the implementation of adequate information and measurement systems. In recent years, there have been various initiatives for improvement in achieving efficient performance and value creation, the special purpose of this research is to propose a model of sustainable system based on indices of Management and Conventional indicators as a result of the study ahead in companies with higher socio-economic impact in the city of Barranquilla and the Caribbean Region.
\end{abstract}

\section{Keywords}

Management, impact, innovation, Oslo Manual, sustainable.

Triana L., (2016). L'innovation financière des entreprises caractérisée par les indices de gestion et les indicateurs conventionnels. Activos, 26, 185-204.

\section{Résumé}

Cet article, résultat de la recherche, analyse lévolution des pratiques et des procédures commerciales à partir du Manuel d'Oslo sur l'innovation, qui 
consiste à mettre en œuvre des systèmes d'information et de mesures appropriés. Ces dernières années ont vu le jour diverses initiatives d'amélioration face à la réussite de la performance et la création de valeur; le but spécial de cette recherche est de proposer un modèle de système durable fondé sur les indices de gestion et les indicateurs classiques, comme résultat de l'étude menée dans des entreprises ayant un impact socio-économique plus élevé sur la ville de Barranquilla et la région des Caraïbes en Colombie.

\section{Mots-clés}

Gestion, impact, innovation, Manuel d'Oslo, durable.

\section{Introducción}

Actualmente, las empresas se configuran hacia la adopción de un sistema sostenible que persigue el mejoramiento de su desempeño en los ámbitos económico, financiero, social y ambiental, con base en estrategias que aborden no solo la calidad de sus productos y servicios, sino también sus procesos con la identificación de los riesgos asociados y las oportunidades, teniendo en cuenta que los cambios en las prácticas y en los procedimientos tienden hacia la optimización de la productividad y el manejo adecuado de los costos de transacción internos para clientes y proveedores, al igual que la introducción de procesos de gerencia de valor y de gestión de la calidad.

El presente artículo, que es producto del proyecto de investigación "Manejo óptimo del capital de trabajo y un adecuado costo de capital como estrategias determinantes de la competitividad empresarial" (registro COLCIENCIAS 043585), propone un modelo de sistema razonable basado en índices de gestión e indicadores que son resultado de estudios que se han adelantado en empresas con mayor impacto socioeconómico en la ciudad de Barranquilla y en la región Caribe, con especial referencia al concepto de innovación empresarial en el contexto de organización y gestión, con la finalidad de determinar si las empresas están siendo exitosas en cuanto a desempeño y resultados. 
Respecto al término innovación, Jansa (2010, p. 55) indica que "los cambios innovadores se realizan mediante la aplicación de nuevos conocimientos y tecnología que pueden ser desarrollados internamente, en colaboración externa o adquiridos mediante servicios de asesoramiento".

Uno de los factores determinantes para mejorar el desempeño empresarial consiste en implementar sistemas adecuados de medición e información que permitan analizar e identificar los principales problemas de las actividades esenciales de las organizaciones: operación, inversión y financiación, siendo el adecuado uso y la aplicación de los indicadores financieros convencionales e índices de gestión las bases para la generación de ventajas competitivas sostenibles y, por ende, de su posicionamiento frente a la competencia nacional e internacional.

La propuesta del modelo integrado de índices de gestión e indicadores convencionales, que permita adelantar los procesos empresariales planteados, se presenta en tres secciones:

\section{Análisis financiero convencional}

Se define como aquel que permite a la gerencia la información necesaria para adelantar una perspectiva de la situación económica y financiera de la empresa, incluido el sector al cual pertenece, con la utilización de procesos prácticos y de reconocido apoyo a la administración, a saber:

- Método estático: Estudio referido a una fecha determinada, el cual muestra la importancia relativa de cada uno de los renglones que componen los estados financieros; como lo indica Pulido (2008, p. 87): "Su cálculo se realiza sobre un momento en el tiempo, permite ver el peso que las diferentes partidas sujetas al estudio tienen sobre una mayor o más relevante".

- Método dinámico: Caracterizado por introducir la variable del tiempo al proporcionar un punto de referencia en la situación de la empresa en un momento dado. Estudio de evoluciones de los diversos elementos de 
los estados financieros que permitan mostrar tendencias y la adopción de medidas correctivas para que los pronósticos que se presenten cumplan los objetivos de desempeño y resultados en la forma lo más óptima posible.

Habitualmente, las organizaciones han efectuado medición de su desempeño basándose en los indicadores clásicos:

- Liquidez

- Nivel de endeudamiento

- Actividad

- Rentabilidad

Los indicadores de liquidez son utilizados para determinar la capacidad que tiene la empresa para resolver obligaciones contraídas a corto plazo, estableciendo que cuanto más elevado es este indicador, mayores posibilidades se tienen para cancelar sus deudas, y cuyos componentes de mayor relevancia se exponen en la figura 1.

Figura 1. Componentes de los indicadores de liquidez

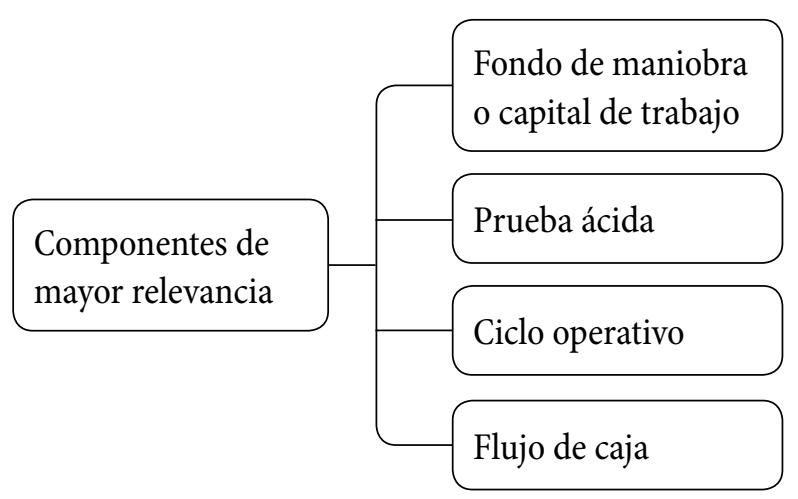

Fuente: elaboración propia.

- El Fondo de maniobra o capital de trabajo se concibe como la parte del activo circulante que es financiada con recursos de carácter transitorio; 
mide la capacidad que tiene la empresa para continuar con el normal desarrollo de sus actividades e indica si una empresa es solvente o no.

- La prueba ácida mide la capacidad de la empresa para cancelar sus obligaciones corrientes, básicamente con saldos del efectivo, cuentas por cobrar, inversiones temporales y algún otro activo de fácil liquidación, sin incluir los inventarios. Para el caso de las empresas de servicios con inventarios muy reducidos, los valores numéricos de la prueba ácida y de la razón corriente son prácticamente iguales.

- El ciclo operativo, como medida de tiempo, está conformado por dos elementos determinantes de la liquidez: conversión de los inventarios y de las cuentas por cobrar Esre ciclo realiza con efectividad la secuencia y relación entre sus procesos y actividades, que permitan controlar y optimizar la información para producir valor agregado y satisfacer la calidad, la productividad y la rentabilidad. Como lo establece Vallejo (2017, p. 21): "Lo lógico en un manejo de cartera y pago a proveedores es vender de contado y pagar a plazos, esto es lo que logran muchas cadenas de supermercados, grandes superficies y muchas multinacionales".

- El flujo de caja muestra las variaciones de entradas y salidas de efectivo, presenta la acumulación neta de activos líquidos y se constituye en indicador imprescindible de la liquidez por la ayuda que presta a inversionistas, proveedores, acreedores y a la gerencia de la empresa en la evaluación de la capacidad para generar flujos de efectivo utilizados para cumplir con las obligaciones contraídas y repartir utilidades.

El nivel de endeudamiento consiste en las deudas contraídas por la empresa relacionadas directamente con sus actividades económicas, dependiendo del nivel de operaciones y su magnitud, cuyo financiamiento espontáneo se deriva en forma natural de la operación diaria, que a su vez está soportado en la estructura financiera como importante elemento de dirección, compuesta por el patrimonio más los recursos financieros que la empresa ha captado u originado y que se clasifican según su procedencia y plazo. COMO lo indica Hamid (2016, p. 102): “Las empresas de América 
Latina están enfrentando condiciones del mercado financiero más difíciles a nivel mundial, en un contexto de menor crecimiento potencial y ajustes macroeconómicos complicados a nivel interno".

La clasificación de las fuentes de financiación empresarial obedece principalmente a tres variables:

- Dependiendo de su origen: internas o externas.

- De acuerdo con la pertenencia: propias o financiación de terceros.

- Según vencimiento: corto y largo plazo.

Figura 2. Clasificación y componentes de las fuentes de financiación

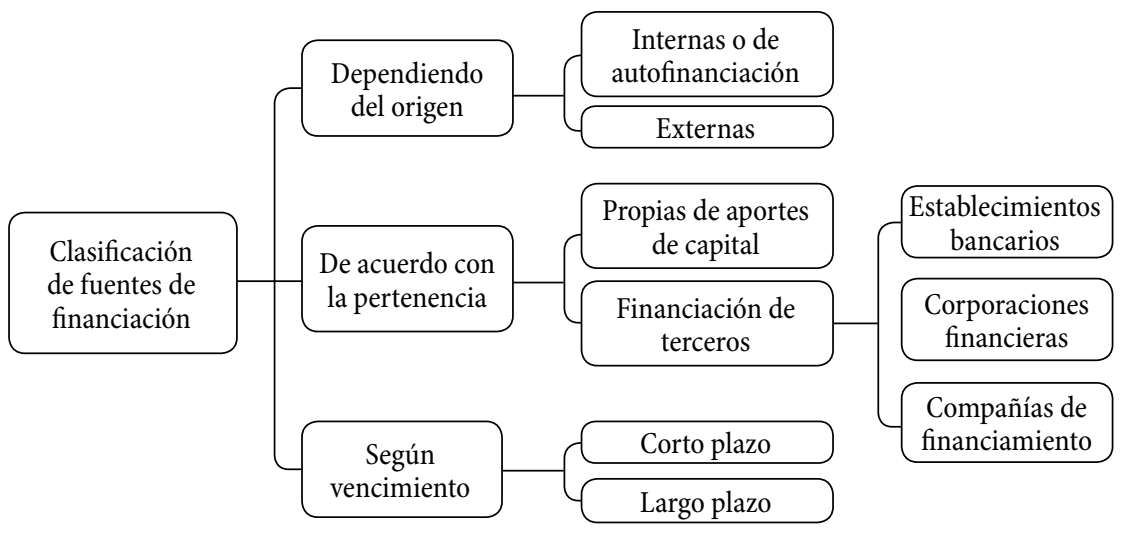

Fuente: elaboración propia.

El manejo óptimo de endeudamiento depende de la estructura de capital, que, a su vez, tiene como objetivo maximizar la riqueza de los accionistas. Dado que la utilización de recursos propios para financiación de la actividad empresarial no es suficiente, las empresas se ven precisadas a la búsqueda y el empleo de fuentes de financiación externas, cometido que requiere una acertada gestión de selección que permita captar recursos al menor costo posible.

- Internas o de autofinanciación, proceden de la propia empresa en forma de reservas, resultados pendientes de aplicar, primas de 
emisión de acciones o de venta de activos no imprescindibles para su funcionamiento.

- Externas, por préstamos de accionistas o socios, las cuales se rigen por lo presupuestado en el Estatuto Tributario en lo relacionado con intereses presuntivos.

- Propias de aportes de capital de socios y accionistas, tanto en la constitución de la empresa como por posteriores ampliaciones, que obviamente conllevan el correspondiente costo de capital, denominados dividendos o distribución de beneficios y que son viables de acuerdo con las expectativas de los propietarios al realizar el aporte sobre el costo de oportunidad.

- Financiación de terceros. Las líneas de crédito en que se enmarcan las operaciones de financiamiento empresarial están contempladas dentro del Estatuto Orgánico del Sistema Financiero, que tiene objetivos claramente definidos en cuanto a supervisión y establecimiento de sistemas de administración de riesgos financieros a nivel consolidado, conformadas especialmente por las siguientes entidades:

- Establecimientos bancarios, con funciones de captación de recursos con el objetivo primordial de realizar operaciones activas de crédito.

- Corporaciones financieras, que según el Estatuto Financiero son aquellas corporaciones que tienen por función principal la captación de recursos a término por medio de depósitos o de instrumentos de deudas de plazo con el fin de realizar operaciones activas de crédito y efectuar inversiones cuyo objeto primordial es fomentar o promover la creación, reorganización, fusión, transformación y expansión de empresas en los sectores que establezcan las normas que regulan su actividad.

- Compañías de financiamiento, captación de recursos del público con el propósito de financiar la comercialización de bienes y servicios y realizar operaciones de arrendamiento financiero o leasing. 
Los indicadores de actividad, también denominados de rotación, tienen el propósito de medir la eficiencia con que la organización utiliza sus activos, teniendo en cuenta que estos deben ser productivos y contribuir al máximo al logro de las metas de la empresa. Surgen del principio elemental de las finanzas según el cual todos los activos de una empresa deben contribuir al máximo en el logro de sus objetivos, es decir, no conviene tener activos improductivos.

Figura 3. Indicadores de actividad o rotación_

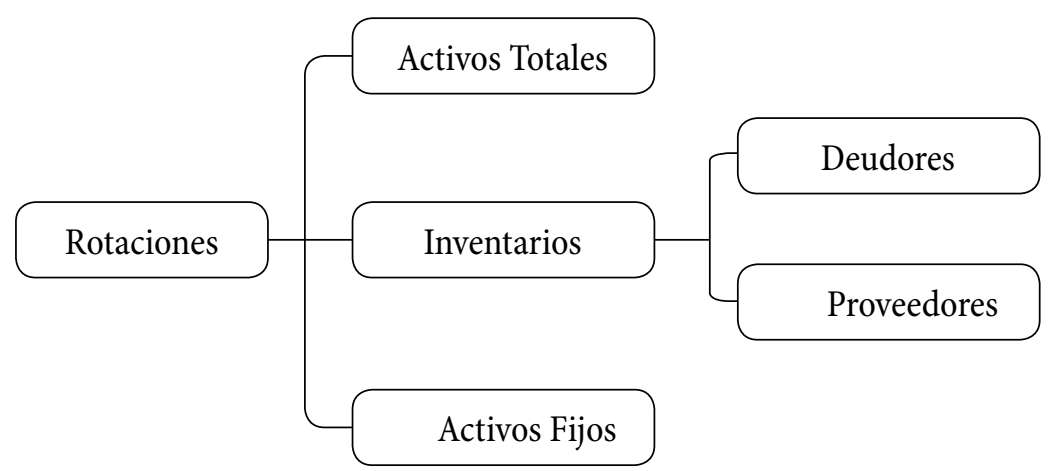

Fuente: elaboración propia.

Estos indicadores se consideran una razón financiera más dinámica porque toman en cuenta información tanto del balance general como del estado de resultados, en búsqueda de cumplir el propósito de las empresas de producir los más altos resultados con el mínimo de inversión.

- Rotación de activos totales: La proporción resultante de este indicador se interpreta comparándolo con el desempeño de períodos anteriores y relacionando la suma total de activos con las ventas generadas. Al respecto, Jhonson (2016) indica que "la rotación de activos totales mide la eficiencia con que las empresas utilizan sus activos para generar ingresos". 
- Rotación de inventarios: Medida utilizada para evaluar la gestión del departamento comercial de la empresa y de control preventivo, que se basa en reposiciones reales ajustadas a las necesidades, evitando así acumulaciones excesivas de stock y que, a su vez, está influenciada por dos aspectos:

- La cartera, cuya gestión debe ser una política de primer orden en la empresa, puesto que de su manejo eficiente depende un acertado capital de trabajo, mejoras de su liquidez e implicaciones que se pueden presentar para poder operar en forma normal o que para el pago a sus proveedores deba recurrir a financiación externa.

- Proveedores, cuyo manejo integral en la actualidad es uno de los ejes estratégicos de la gerencia moderna para la generación del valor agregado por ser el primer paso en la cadena de suministros que refleja su manejo eficiente y mejoras en competitividad al establecer un plan de requisiciones que debe ejecutarse de acuerdo con las condiciones establecidas de calidad, costo, plazo y servicio posventa.

- Rotación de activos fijos: En la actualidad, debido principalmente a la implantación de las NIIF y a la dinámica de los negocios, leyes y procedimientos, se ha generado la necesidad de administrar la cuenta de activos fijos de manera confiable y eficiente, ya que en el pasado su control se limitaba solo al registro de las adquisiciones y a su natural tratamiento fiscal y contable.

Los conceptos sobre precio de mercado del bien y costo histórico -si bien muestran valores pasados, la exactitud en sus registros y la facilidad de verificación- están siendo substituidos por valor razonable, por dar este mayor relevancia y fiabilidad a los estados financieros. Al respecto, Zúñiga, Pacheco y Díaz (2009, p. 76) afirman que el valor razonable, según lo define IASB en su NIC 16, corresponde al "importe por el cual podría ser intercambiado un activo, o cancelado un pasivo, entre partes interesadas y debidamente informadas, en una transacción realizada en condiciones de independencia mutua". 
Indicadores de rentabilidad. El análisis de los niveles de rentabilidad de una empresa establece que la información financiera contenida en los estados financieros debe reunir características de variables cuantitativas y cualitativas que permitan asegurar el cumplimiento de suministrar información razonable, tanto interna como externa.

Figura 4. Indicadores de rentabilidad

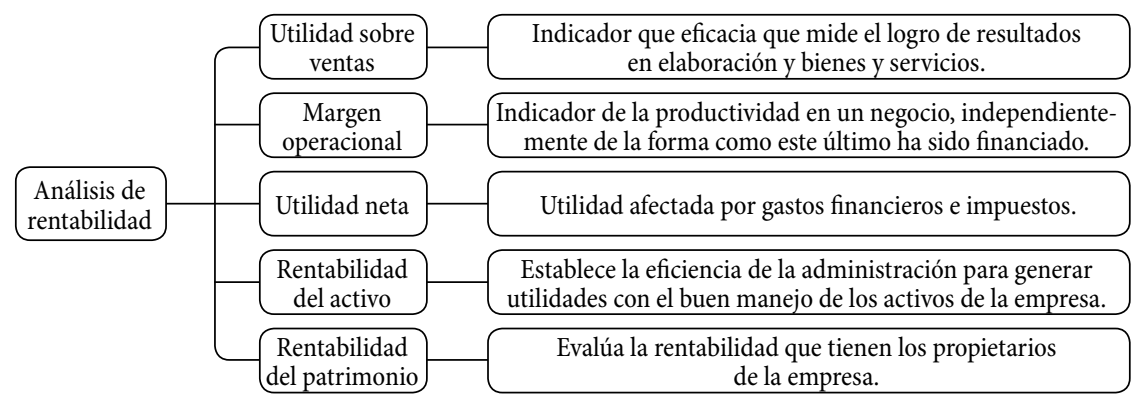

Fuente: elaboración propia.

- Variable cuantitativa, relacionada con una revisión detallada consistente en una serie de pruebas matemáticas y numéricas que pueden servir como parámetro para diagnosticar el perfil financiero de la empresa, teniendo presente el principio de que cualquier inversión debe ser autosuficiente para mantener su valor e incrementarla.

- Variable cualitativa, cuyas características esenciales son:

- Confiable, veraz, representativa, objetiva y verificable.

- Degran relevancia, con alta posibilidad de predicción, confirmación e importancia relativa.

- Comprensible, para que la información sea entendida por los usuarios.

- Comparable con análisis sobre identificación de diferencias y similitudes con otras entidades del sector a través del tiempo. 


\section{Índices de gestión}

La innovación financiera y la gerencia de valor requieren de nuevas estrategias que complementen el análisis convencional, conformando así un diagnóstico financiero integral que se construye con información, además de los estados financieros, el flujo de caja y otras fuentes externas de valoración de mercados mediante análisis y seguimientos mucho más amplios que incluyan variables no solo de orden cuantitativo, sino en especial cualitativo, cuya objetivo primordial está orientado a la valoración de empresas. Al respecto, Triana (2015, p. 45) expresa:

La valoración de empresas es, en la práctica, el principal instrumento financiero actualizador, ya que debido al permanente cambio en las oportunidades y tendencias de los negocios, al alto nivel de incertidumbre, complejidad y competitividad empresarial, se hace indispensable conocer objetivamente el verdadero valor de una organización.

Dentro del diseño de índices de gestión, en la actualidad y como necesidad ineludible para desarrollar una cultura orientada hacia el aprovechamiento de los recursos de una organización logrando su eficiencia y eficacia, se hace necesario contar con un sistema de índices cuyos principales componentes se hallan en:

E. V. A. (valor económico agregado). Comprende análisis del costo de capital, expresado como la tasa de rendimiento que debe ganar la empresa para incrementar su valor, el cual está representado por el retorno de capital de quienes financian a la empresa: proveedores, mediante diferentes medios (préstamos, leasing, bonos), e inversionistas (adquisición de acciones). Solé (2016, p. 28) define el E. V. A. como la "herramienta que permite calcular y evaluar la riqueza generada por la empresa, tomando en consideración el nivel de riesgo con el que opera”. 
Figura 5. Estructura óptima de capital y el E. V. A.

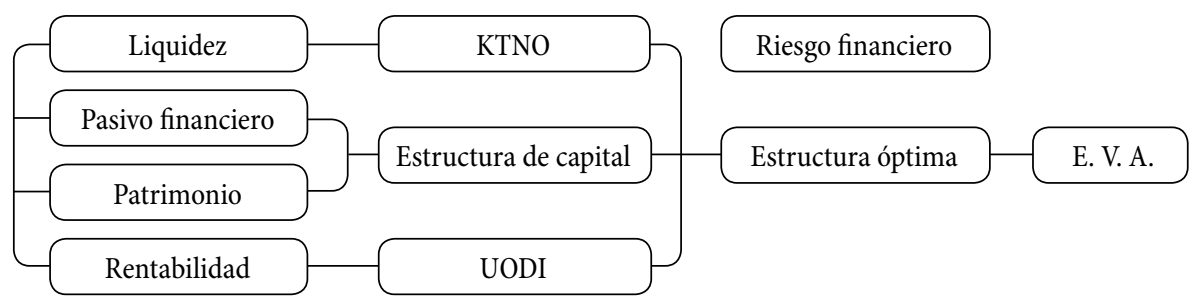

Fuente: elaboración propia.

Tradicionalmente, los resultados contables se consideraban suficientes para la gestión empresarial y la toma de decisiones, sin embargo, debido a la globalización y competitividad actuales, se otorga mayor importancia al conocimiento y a la aplicación de medidas tendientes a la creación de valor y a la capacidad de gestionar dicha creación, con la utilización de la estructura óptima de capital. Dussan (2016, p. 34) afirma que "si el valor económico agregado es positivo, significa que la empresa ha generado una rentabilidad por arriba de su costo de capital, lo que le genera una situación de maximización del valor de la unidad empresarial".

Se considera como estructura óptima de capital de una empresa el análisis de información que permita una evaluación integral de la inversión, financiación y el riesgo financiero, los cuales comprenden:

- KTNO: En el aspecto financiero, se tiene la percepción de suponer el capital de trabajo neto operativo como la cartera, inventarios y la financiación de proveedores, que permiten determinar cuánto debe mantenerse en capital de trabajo por cada peso generado en ventas, y acorde con dicha perspectiva, garantizar el mayor volumen de ventas posible con el menor capital invertido.

- UODI: Utilidades operacionales después de impuestos, medida en la cual no se toman en consideración los gastos por intereses que dependen de la estructura de capital de la empresa y otros gastos que no son propiamente producto de la operación normal, permitiendo 
contrastar con mayor precisión la utilidad después de impuesto de renta y compararse con otras empresas del sector, esto con el fin de evaluar la verdadera gestión y la más eficaz.

- Riesgo financiero: Referido a la probabilidad asociada de que un evento pueda tener consecuencias financieras negativas, con posibilidades de que los beneficios por una inversión sean menores que los esperados o la posibilidad de que las expectativas positivas para un sistema orientado al logro de objetivos no se realicen.

- EBITDA:Earnings before interests, taxes, depreciations and amortizations. Indicador de gran beneficio para la empresa, utilizado como referencia de suactividad operativa; muestralos resultados antes de intereses, impuestos, depreciaciones y amortizaciones, y señala que la administración de operaciones debe incluir múltiples actividades relacionadas con cambios en el ambiente socioeconómico y, en términos generales, todos aquellos que les son propios a la organización y su entorno.

Figura 6. Elementos constitutivos del EBITDA

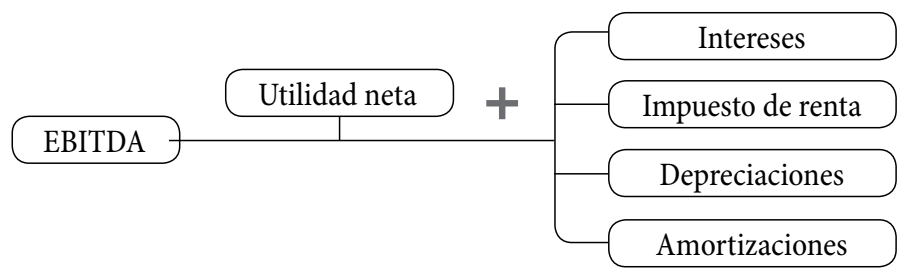

Fuente: elaboración propia.

A pesar de no formar parte del estado de resultados, se constituye en un indicador muy utilizado como referencia sobre la capacidad de una empresa para generar beneficios considerando únicamente su actividad productiva, ya que, como lo indica Jiménez (2015, p. 20):

Se suele tener en cuenta cuando se quiere analizar la generación operativa de negocios de una empresa cuando estos requieren una importante inversión, puesto que son aquellos los que más amortizaciones deben registrar, falseando estas en cierta manera el resultado real. 
F. C. L. (flujo de caja libre). Informe financiero de mayor actualidad que permite medir la capacidad de la empresa para atender los costos de financiación, por intereses y/o dividendos, como resultado de la operación normal, presentando en detalle ingresos y egresos en efectivo.

Este índice de gestión, que tiene varias interpretaciones, sobresale en la evaluación de los negocios desde el punto de vista de los inversionistas y financistas de la empresa, y es el punto de partida para saber si una empresa es viable en su operación o no. Al respecto, Bedoya (2012, p. 90) indica: “Si asumimos un flujo de caja constante, el valor de una empresa como negocio en marcha es igual a dicho flujo de caja dividido por la tasa de oportunidad".

Figura 7. Variables de la estructura del flujo de caja libre

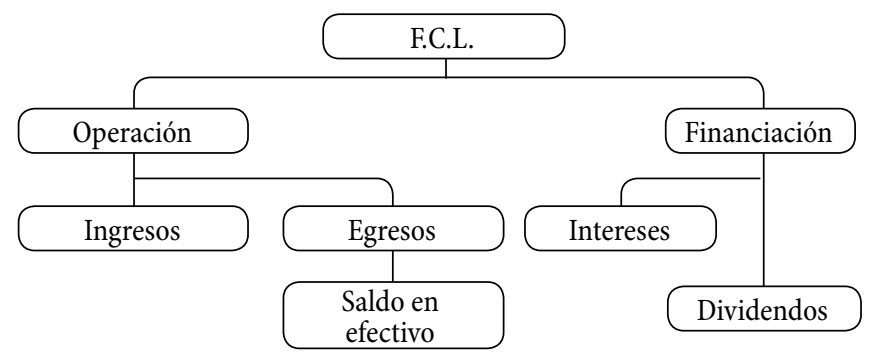

Fuente: elaboración propia.

El flujo de caja libre (cash flow) revela cuánto genera la empresa por sus operaciones; y por tratarse de una integración del valor agregado y el EBITDA, en el caso de los proyectos de inversión, para el cálculo de los flujos de caja futuros se toman como base los estados financieros proforma, el análisis de KTNO y las proyecciones estimadas de ventas y de variables macroeconómicas -como la DTF, la inflación, TIR y VPN- con el fin de determinar la inversión de activos productivos que garanticen la continuidad del negocio.

Neffa (2014, p. 39) expresa:

No siempre que una empresa genera un flujo de caja negativo es malo, porque puede estar creciendo y necesitando de saldos de caja para poder cubrir sus necesidades de inmovilización de dinero en activos fijos o de 
incremento del capital de trabajo, como es el caso cuando las empresas acumulan acciones previendo mayores ventas a futuro.

\section{El desarrollo de un modelo de innovación financiera}

Como resultado de los anteriores conceptos y definiciones, se puede concluir que la innovación financiera, considerada como uno de los factores básicos de desarrollo, tiene como finalidad ayudar a prever las necesidades del mercado, y para ello recurre a procesos y servicios de mayor calidad, genera rentabilidad con un manejo adecuado de costos, implementa un sistema apropiado de indicadores en posiciones estratégicas que reflejen las mediciones de la gestión y que evidencien buenos resultados, todo mediante un modelo de información integrador que permita medir las diferentes etapas del proceso.

Para la construcción del modelo, se identifican tres instrumentos que, de acuerdo con sus funciones y manejo de los riesgos, permiten la generación de liquidez, fuentes de financiación, inversión y capital, como son los que se identifican a continuación: valor agregado, beneficio operacional desembolsable y capacidad financiera.

Figura 8. Modelo sostenible de innovación financiera

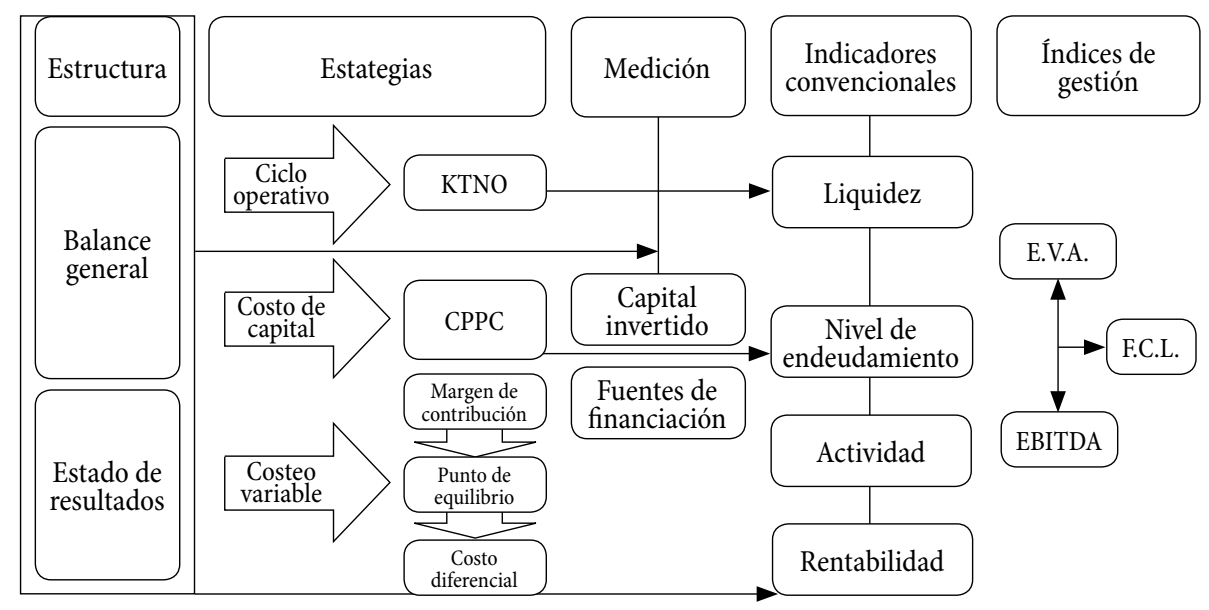

Fuente: elaboración propia. 
Se expone a continuación un caso práctico de la Empresa Comercializadora $\mathrm{ABC}$ S. A.:

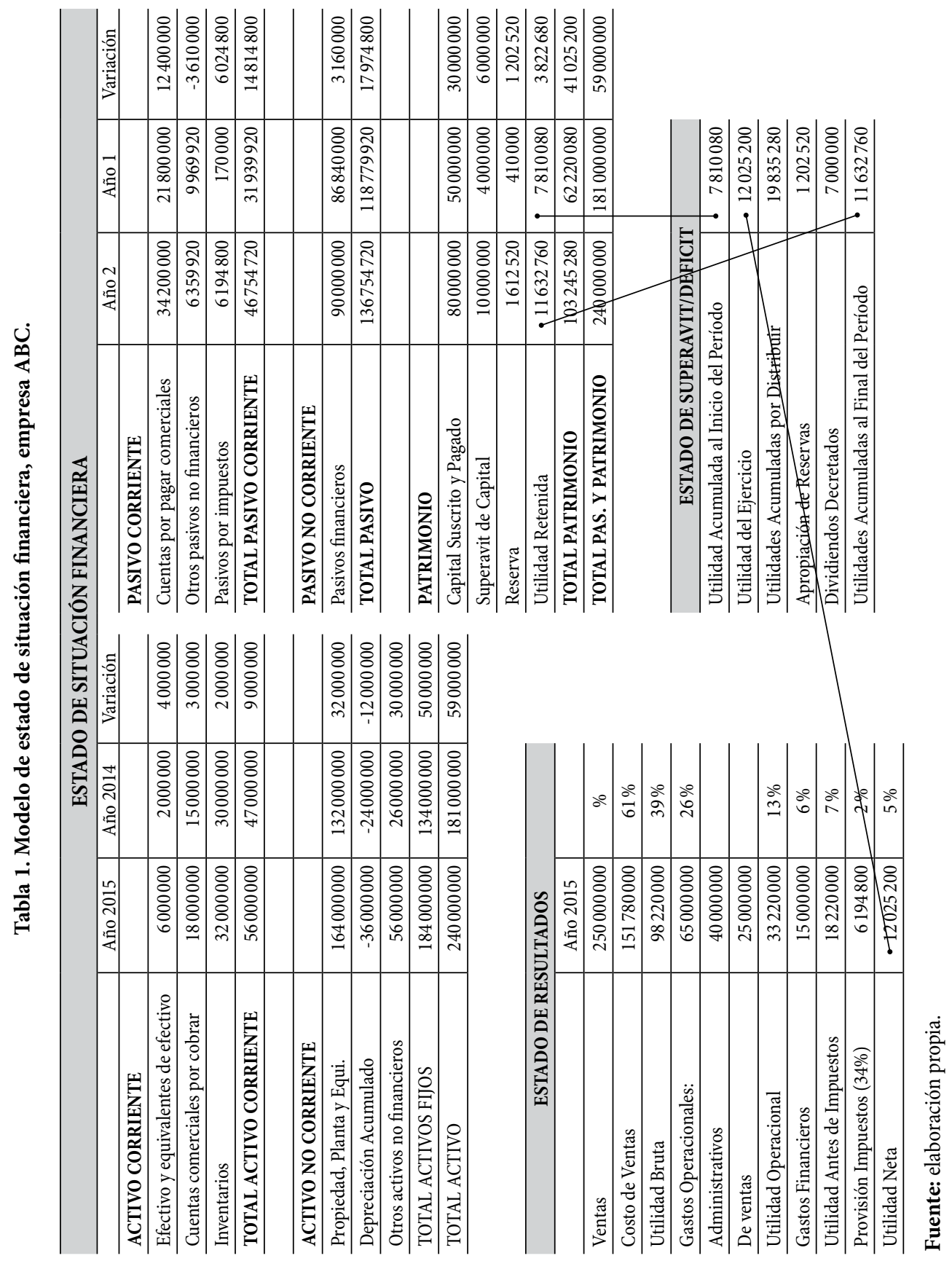




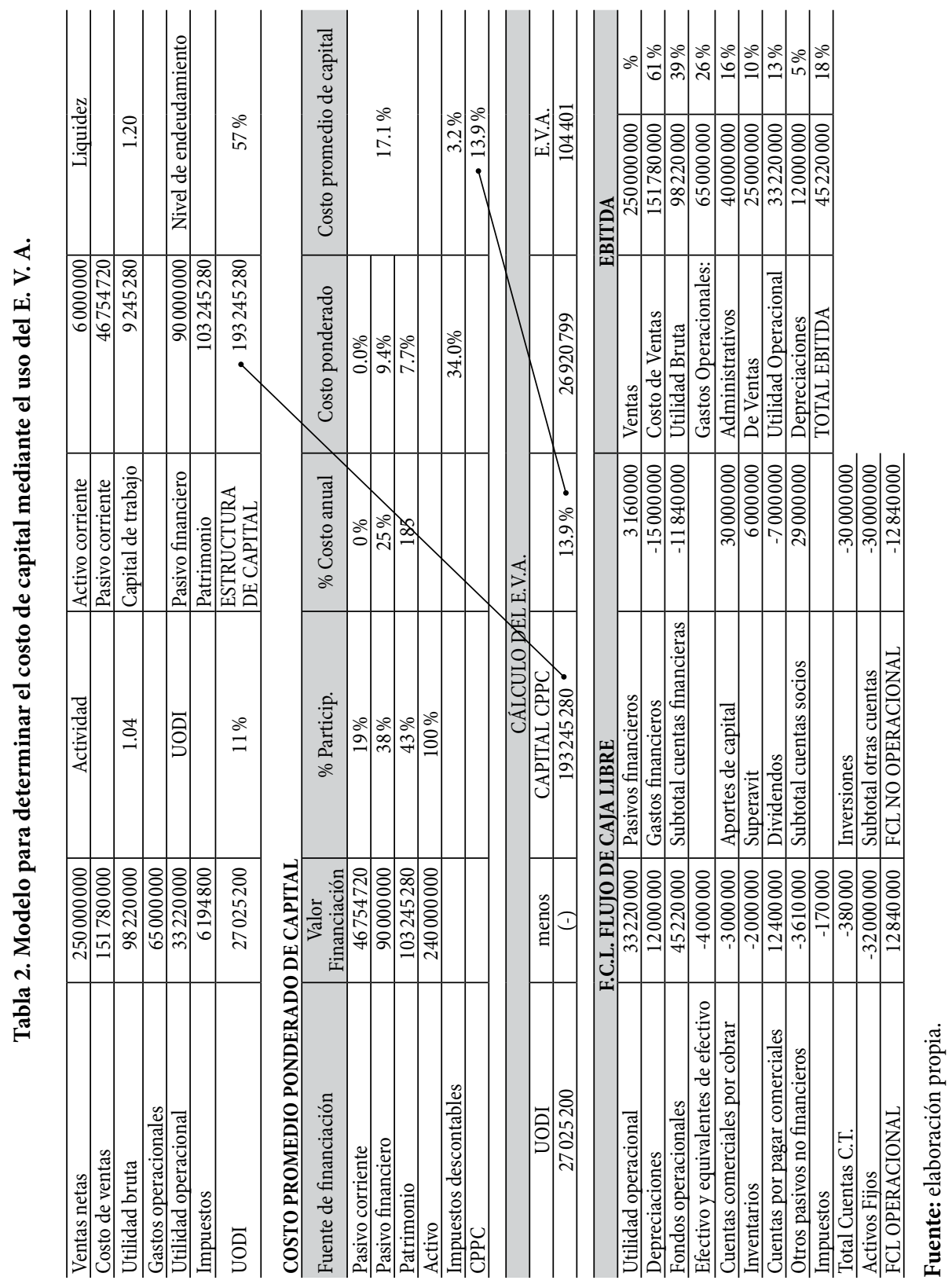




\section{Interpretación:}

- El E. V. A. de \$104.401, como resultado positivo, indica que la empresa está agregando valor al capital inicialmente aportado y generando mayores rendimientos a los accionistas, por lo tanto, es viable en su operación y financiación.

- En caso negativo, se revelaría que los rendimientos son menores que lo que cuesta generarlos y, por lo tanto, se estaría destruyendo riqueza para los accionistas, es decir, estarían en una situación más favorable si hubieran elegido otras alternativas de inversión de igual riesgo.

- El EBITDA representa un $5 \%$ adicional a la utilidad operacional de $13 \%$, lo cual permite mostrar una utilidad depurada que proporciona idea clara del rendimiento operativo de las empresas, haciendo posible comparar la rentabilidad en el contexto interno y externo.

- El F. C. L. positivo proporciona información vital para poder interpretar la viabilidad de la empresa, tanto en la liquidez como en el manejo operativo y de inversiones, además de poder atender normalmente los gastos por intereses y distribución de dividendos.

La interpretación de un flujo de efectivo negativo es señal preocupante acerca de la liquidez y un claro aviso de un excesivo nivel de endeudamiento.

\section{Conclusiones}

De acuerdo con los resultados obtenidos en el uso de análisis financiero tradicional que utilizan las empresas y las necesidades actuales de crear guías de actividades concretas consideradas de innovación, como lo plantea el Manual de Oslo, y con la finalidad de reunir e interpretar datos que determinen si la gerencia está siendo exitosa en cuanto a desempeño y toma de decisiones eficientes, se propone el modelo sostenible de innovación financiera, recomendado para unificar los indicadores habituales y los índices de gestión E. V. A., EBITDA y F. C. L., con el fin de implementarlo 
en organizaciones de todo tipo, con el propósito de que puedan realizar sus actividades en forma eficiente, eficaz y con calidad.

\section{Referencias bibliográficas}

Bedoya, J. (2012) Diez conceptos sobre el flujo de caja libre. Recuperado de goo. $\mathrm{gl} / \mathrm{XlWo3O}$

Dussan, C. (2016) Gerencia financiera. ¿Qué es el EVA y el CVPPC? Recuperado de goo.gl/eri7Iw

Hamid, F. (2016). Perspectivas económicas. Las Américas. Administrando transiciones $y$ riesgos. Washington: Ed. Fondo Monetario Internacional. Recuperado de goo.gl/2udHEC

Jansa, S. (2010). Resumen del Manual de Oslo sobre innovación. Recuperado de goo.gl $/ 6 \mathrm{mP} 23 \mathrm{~B}$

Jiménez, C. (2015).¿Quées el EBITDA y por qué es tan buen indicador de resultados? Recuperado de goo.gl/oGtQJo

Johnson, R. (2016). Las definiciones de rotación de activos totales y el margen de ganancias (Trad. D. L. Arjones). Recuperado de goo.gl/RN7DZ8

Neffa, G. (2014). El flujo de caja para interpretar si una empresa hace bien las cosas. Recuperado de goo.gl/XeOCbY

Pulido, A. (2008). IEDGE - El análisis financiero estático o vertical. Recuperado de goo.gl/XLP5Ua

Solé, R. (2016). Valor económico agregado (EVA). Recuperado de http://finanzaslpucr-sole.blogspot.com.co/2016/06/sesion-14-valor-economico-agregado-eva. html

Triana, L. (2015). Nuevas tecnologías para valoración de empresas en el sector turístico. En B. H. Díaz-Solano (Coord.), Perspectivas y prospectivas del desarrollo turístico en la zona costera del departamento del Atlántico (pp. 177-208). Barranquilla: Ed. Uniautónoma.

Vallejo, P. (2016). Cómo acelerar el ciclo operacional de su empresa. Recuperado de goo.gl/B4XBP5

Zúñiga, F., Pacheco, L. y Díaz, J. (2009). Convergencia contable: cambios profundos en la contabilidad chilena. Activo fijo, un caso a considerar. CAPIC REVIEW, 7, 75-82. Recuperado de goo.gl/dkVcpP 